\title{
SOC NANOBASED INTEGRATED WIRELESS SENSOR SYSTEM
}

\author{
Penghua Sun, Maher Rizkalla, and Mohamed El-Sharkawy \\ Purdue School of Engineering and Technology, Indianapolis, Indiana, USA
}

\begin{abstract}
Smart nanotechnology materials have been recently utilized in sensing applications. Carbon nanotube (CNT) based SoC sensor systems have potential applications in various fields, including medical, energy, consumer electronics, computers, and HVAC (heating, ventilation, and air conditioning) among others. In this study, a nanotechnology multisensory system was designed and simulated using Labview Software. The mathematical models were developed for sensing three physical quantities: temperature, gas, and pressure. Four CNT groups on a chip (two for gas sensor, one for temperature, and a fourth one for pressure) were utilized in order to perform sensing multiple parameters. The proposed fabrication processes and the materials used were chosen to avoid the interference of these parameters on each other when detecting one of them. The simulation results were translated into analog voltage from Labview software, transmitted via Bluetooth network, and received on desktop computers within the vicinity of the sensor system. The mathematical models and simulation results showed as high as $95 \%$ accuracy in measuring temperature, and the 5\% error was caused from the interference of the surrounding gas. Within 7\% change in pressure was impacted by both temperature and gas interference.
\end{abstract}

\section{INTRODUCTION}

\subsection{Nanosensors}

Nanotechnology has recently explored unique features related to smart nanomaterials such as fullerene, carbon nanotube, graphene, quantum dots, nanophotonic crystals, magneto resistance material, and nano polymers [1]. Figure 1 shows the image of the Fullerene, quantum dots and CNTs, that can sense physical quantities such as temperature, pressure, gas, and magnetic fields by changing their electrical or magnetic properties. They can change their conductivity when exposed to a gas. The capacitance of the device will also change when the CNTs are exposed to these physical quantities [2-3]. There are mainly three types of CNTs: Single-walled (SWCNT), double-walled (DWCNT) and multi-walled (MWCNT) as shown in Figure 1. Single-walled CNTs can be seen as a closed-loop of graphene (diameter of about 1 nanometer), while doublewalled and multi-walled CNTs are basically several single-walled ones placed in a coaxial configuration (diameter of about 10s of nanometers) [4]. Reference [5] discussed the design of a radio frequency (RF) based system that detected the change in the reflected RF power due to the change in the device capacitance, and accordingly, to the exposed gas. The fabrication of the CNTs may be demonstrated by separation and enrichment [6].

David C. Wyld et al. (Eds) : SAI, CDKP, ICAITA, NeCoM, SEAS, CMCA, ASUC, Signal - 2014 pp. 333-342, 2014. () CS \& IT-CSCP 2014

DOI : $10.5121 /$ csit.2014.41130 


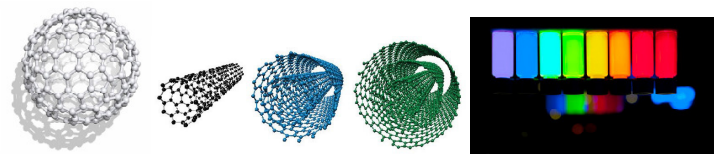

Figure 1: Image of Fullerene, Quantum Dots and CNTs.

Researchers have succeeded to implement nanotechnology sensors that sense one parameter such as temperature, gas, or pressure. There is a need, however, to have multiple sensors on a chip that can detect a combination of parameters simultaneously to perform multiple bio functions. Since the CNT material changes with all parameters when exist simultaneously, it is hard to detect the impact of each parameter on the CNT based sensor. The exposure of the CNT device to both temperature and gas present in the same chamber are conducted. The resulting change in the resistance cannot precisely predict the change of each, separately. Furthermore, the change in the pressure in the presence of the temperature and gas may not reflect the true value of the pressure quantity. A reliable system should resolve the interference in order to separately detect the impact of every parameter separately. Therefore, building three sensors in the same chip, each to detect one quantity separately is quite challenging since the three sensors are set into the same chip and exposed to all parameters' changes. Other issues may include the type of assembly of the CNT materials that may be chosen to provide the range of temperature, gas, or pressure to be suitable for Bio-applications.

\subsection{Processing Unit}

An interface unit may be necessary to provide the power requirement to drive the processing unit. A signal conditioning unit may be needed to provide the current levels suitable for driving the output stages. A nanoelectromechanical system (NEMS) may be implemented on board with the processing unit to provide data analysis and wireless transmission. The processing unit may take advantage of the available hardware board system associated with the Labview software. This research approach accommodated each sensor separately to sense one quantity. This was accomplished by shielding it from the interference of other quantities. The small percentage error can be incorporated in the mathematical model of the processing unit.

\section{SYSTEM DIAGRAM}

The temperature and pressure sensor system may be utilized in bioengineering applications, including electrocardiography (ECG), human temperature, and heart rate, among others. Figure 2 shows a general medical setting for the use of multiple sensor system via wireless network. 

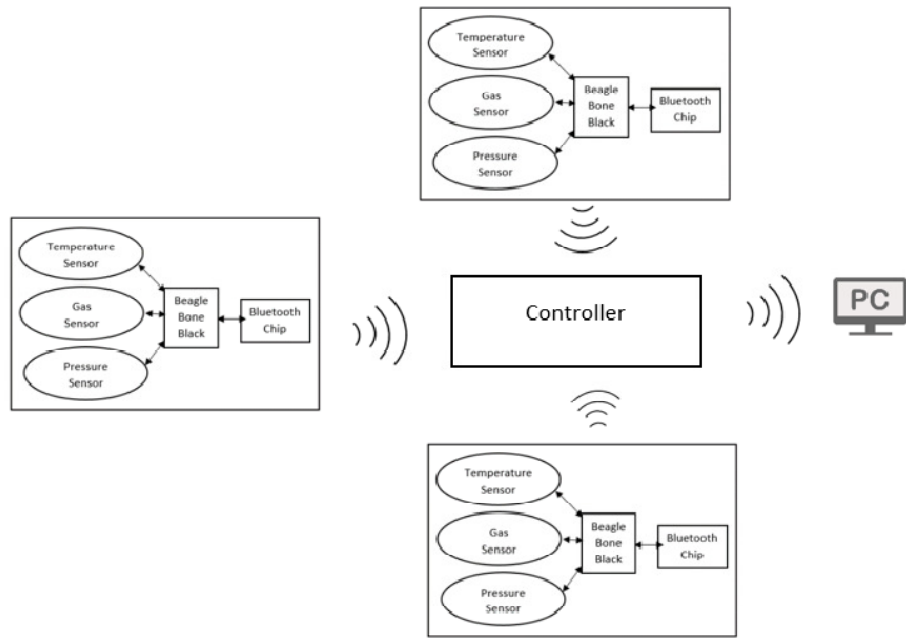

Figure 2: System Diagram.

\section{SENSOR MODELS}

\subsection{The Pressure Sensor}

The pressure sensor designed for this system was based on detecting the change in conductivity caused by pressure, resulting in a change of the material resistance. The substrate material selected for this purpose was chosen based on its linearity and pressure coefficients. PMMA (polymethyl methacrylate is a versatile polymeric material that is well suited for many imaging and non-imaging microelectronic applications. It is a common positive resist for e-beam, x-ray, and deep UV microlithographic processes) substrate material was found to be appropriate for this design [7]. Figure 3 gives the shape of the device. The detection of a clamped circular shape under a uniform pressure $\mathrm{P}$ is denoted by $\mathrm{w}$ and is given by [8]:

$$
\mathrm{w}=\frac{\mathrm{p} \mathrm{a}^{2}}{264 \mathrm{D}}\left[1-(\mathrm{r} / \mathrm{a})^{2}\right]^{2}
$$

Where $r$ and a are the radial coordinate and diaphragm radius, respectively. It is clear from Figure 3 that $r$ is the original radius and $a$ is the radius change. $D$ is a measurement of stiffness and is given by:

$$
D=\frac{\mathrm{E} h^{3}}{12\left(1-\mathrm{v}^{2}\right)}
$$

Where E, h, and v are Young's modulus, plate thickness, and Poissons ratio, respectively. 


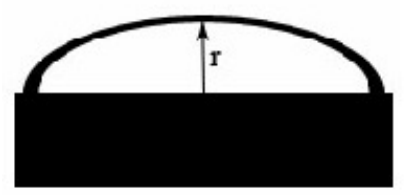

Figure 3: The Shape of PMMA Device.

The room temperature resistance at $0 \mathrm{kPa}$ of a sensor was typically ranged from several $\mathrm{k} \Omega$ to several hundred $\mathrm{k} \Omega$. Some works have been done previously [9] showed that the resistance across the CNTs increases linearly with applied pressure up to $70 \mathrm{kPa}$. Figure 4 gives the pressuredetection curve, showing the effect of the diameter size of the substrate
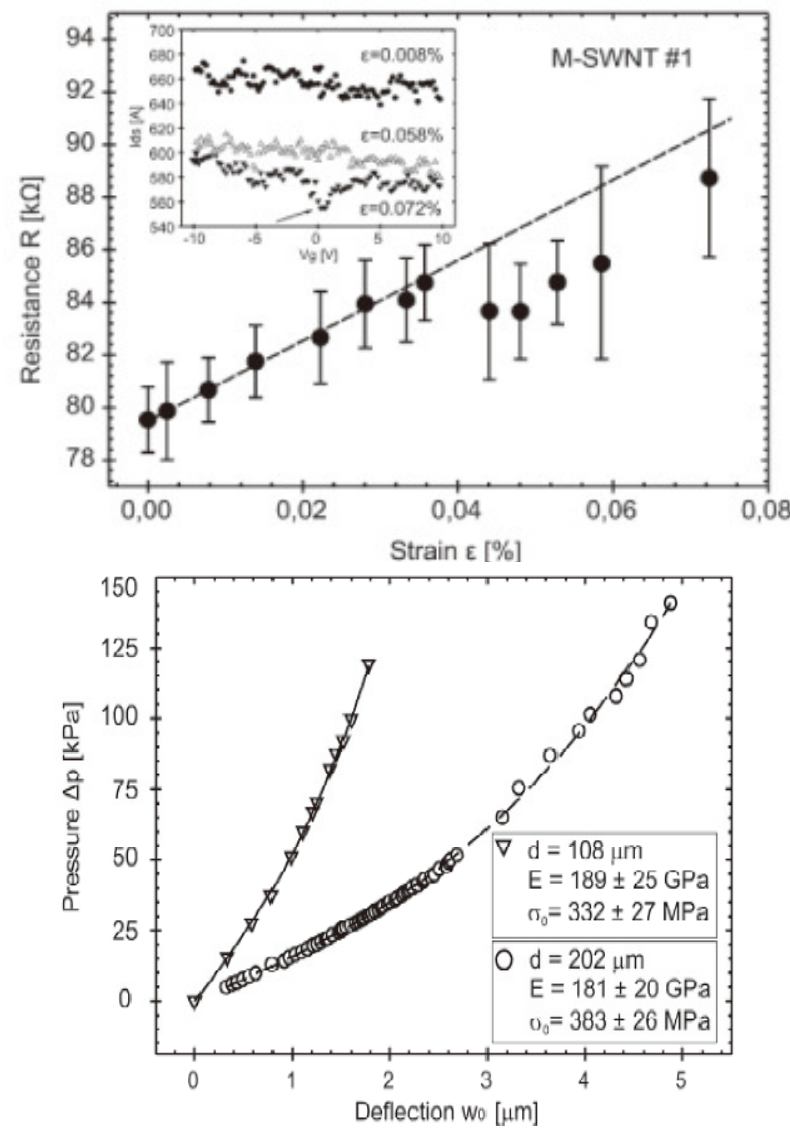

Figure 4: The Pressure/Deflection and Strain Curves.

\subsection{Resistance Model}

The gauge factor of the CNT is given by [2]:

$$
G=\frac{\Delta R}{R}\left(\frac{1}{\varepsilon}\right)
$$


Where $\mathrm{R}$ and $\Delta \mathrm{R}$ are the initial resistance of the sensor before the pressure is applied, and the resistance change of the CNT under the pressure, respectively, and $\varepsilon$ is the strain of the sensor material.

The resistance as function of the strain parameter is given by [3,4]:

$$
R(\varepsilon)=R_{0}+\tilde{R}_{1} \varepsilon
$$

In this expression, $\mathrm{R}_{0}$ is the resistance without strain, $\tilde{R}_{1}$ is the strain coefficient. The model with the strain coefficient is given by [5]:

$$
R(\varepsilon)=R_{S}+\frac{1}{|t|^{2}} \frac{h}{8 e^{2}}\left[1+\exp \left(\frac{\tilde{E}_{g} \varepsilon}{k_{B} T}\right)\right] \approx\left(R_{S}+\frac{1}{|t|^{2}} \frac{h}{4 e^{2}}\right)+\left(\frac{\tilde{E}_{g}}{|t|^{2}} \frac{h}{8 e^{2} k_{B} T}\right) \varepsilon
$$

Where $R_{S}$ is the series resistance of the junction due to SWCNT metal contacts, $|t|^{2}$ is the transmission through the nanotube, and $E_{g}=\widetilde{E}_{g} \varepsilon$ is the strain-dependent band gap for metallic nanotubes, neglecting the torsion contributions. Figure 4 shows a typical resistance-stain curve, showing the effect of the diameter size in the pressure range, leading to the proper deflection that may be detected by the processing unit.

The sensitivity of CNT to detect pressure changes was found to be $54 \mathrm{pA} / \mathrm{mbar}$ for three different strain coefficients averages [6]. Figure 5 shows the sensitivity curve considered for the design of the pressure sensor. Heartbeat pressure is about 170mbar, and the current change is about 10nA current change $(\mathrm{Vds}=200 \mathrm{mV}, \mathrm{Vg}=4 \mathrm{~V})$, which make the design of the sensor system appropriate for bio-engineering applications. This shows the appropriate sensitivity of the selected material.

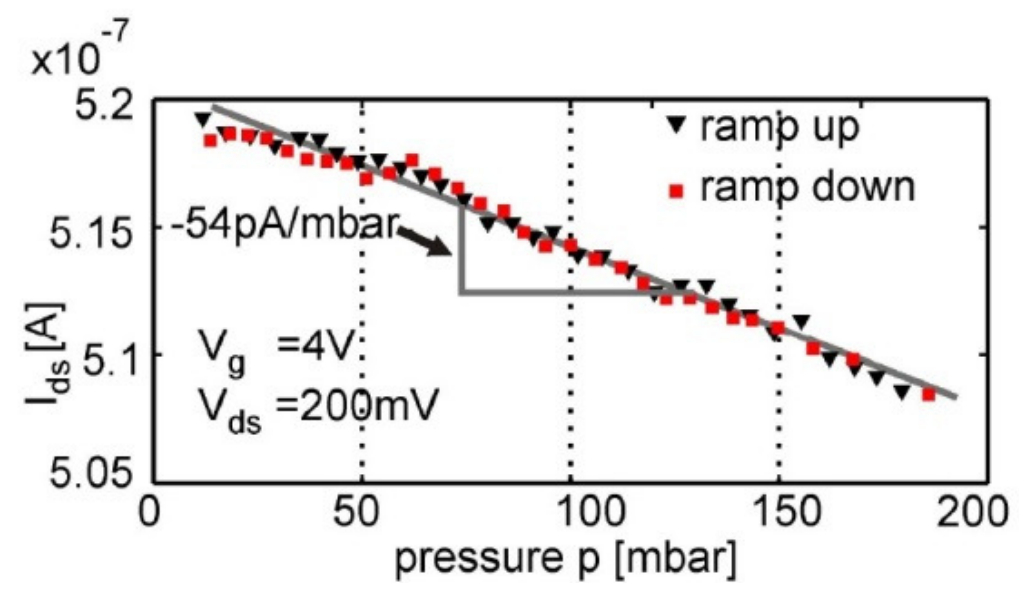

Figure 5: The Sensitivity Curve of Pressure Sensor.

As it can be seen from the data information of this sensor, the sensor is appropriate to measure up to 200 mbar linear range. The minimum current observed was near 200 mbar at $0.5 \mu \mathrm{A}$, which is still appropriate to be processed by the hardware processing unit. 


\subsection{The Temperature Sensor Model}

\subsubsection{The Concept}

The concept of the temperature sensor is based on exhibiting the change in the CNT resistance material when exposed to change in temperature. The temperature change may also alter the pressure, and the thermal expansion (or contraction) of the Polyamide material.

\subsubsection{The Device Model}

When the temperature changes, the polyamide alters its volume, and accordingly, the pressure from it to the CNTs sensing material. Then the CNTs will exhibit a change in resistance that can be detected, and the change will be processed by the DSP unit, producing the pressure data in the form of electrical energy. The model of the pressure sensing element of the temperature sensor is the same as the pressure sensor model. Thermal stress pressure can be written as $\Delta P=E \alpha \Delta T$, Where $\mathrm{E}$ is young's modulus which is near $1.1 \mathrm{GPa}$ for polyamide, and $\alpha$ is the thermal expansion coefficient and it is about $110 \times 10^{-6} \mathrm{~m} / \mathrm{mK}$ for the same material.

\subsection{The Gas Sensor Model}

\subsubsection{The CNT Schottkey Model}

A Schottky barrier diodes (or MS diodes) are characterized by the so-called Schottky barrier height (SB), and denoted by the metal semiconductor potential barrier $\Phi_{\mathrm{B}}$. In our model, the CNTs were semi conductive SWCNT and the base is metal. When the CNT exposed to the gas, such as $\mathrm{NO}_{2}$, the $\mathrm{S}_{\mathrm{B}}$ height increases, following the model given in [4], and [5], and given below for a P-channel device:

$$
\Phi_{B}=\left(\Phi_{C N T}+\frac{E_{g}}{2}\right)-\Phi_{M}
$$

Where $\Phi_{C N T}=4.7 \mathrm{eV} . \quad E_{g}$ and $\Phi_{M}$ are the band gap and metal work function respectively. For the nanoscale devices such as in CNTs, the potential barrier as determined by [6] is modeled as:

$$
\Phi_{S B} \approx \frac{k T}{\beta} \ln \left(\frac{\alpha \sqrt{\frac{E g}{2 k T}}}{\ln \alpha \sqrt{\frac{E g}{2 k T}-\frac{\Phi_{B}}{k T}}}\right)
$$

In the above expression, $\alpha=\frac{e^{2}\left(\frac{2}{\pi}\right)^{\frac{3}{2}}}{3 \sqrt{\beta} \alpha \gamma d C} \quad(\beta=0.7, \alpha=0.142 \mathrm{~nm}$ C-C bond length, $\mathrm{d}=\mathrm{CNT}$ diameter, $\gamma=2.5 \mathrm{eV}$ is tight-binding overlap integral, $\mathrm{e}=$ electron charge, $\mathrm{k}=$ Boltzmann constant, $\mathrm{T}=300 \mathrm{~K}$, and $\mathrm{C}=$ capacitance per unit area between metal-CNT). Assuming that $\Phi_{S B . i}$ and final $\Phi_{S B . f}$ are the initial and final value of the SB height before and after the gas adsorption, respectively, with the assumption that the band gap of the CNT does not change after gas, the $\exp \left[\frac{\Delta \Phi_{S B}}{k T}\right]$ term can be written as:

$$
\exp \left[\frac{\Delta \Phi_{S B}}{k T}\right]=\exp \left[\frac{\Phi_{S B . f}-\Phi_{S B . i}}{k t}\right]
$$


Combining equations 6 and 7, we get,

$$
\begin{aligned}
& \exp \left[\frac{\Delta \Phi_{S B}}{k T}\right]=\exp \left[\frac{\Phi_{S B . f}-\Phi_{S B . i}}{k t}\right]=\exp \left[\frac{1}{\beta} \ln \left(\frac{\alpha \sqrt{\frac{E g}{2 k T}}}{\ln \alpha \sqrt{\frac{E_{g}}{2 k T}}-\frac{\Phi_{B . f}}{k T}}\right)-\frac{1}{\beta} \ln \left(\frac{\alpha \sqrt{\frac{E_{g}}{2 k T}}}{\ln \alpha \sqrt{\frac{E_{g}}{2 k T}}-\frac{\Phi_{B . i}}{k T}}\right)\right]= \\
& \exp \left[\frac{1}{\beta} \ln \left(\frac{\ln \alpha \sqrt{\frac{E_{g}}{2 k T}}-\frac{\Phi_{B . i}}{k T}}{\ln \alpha \sqrt{\frac{E g}{2 k T}}-\frac{\Phi_{B . f}}{k T}}\right)\right]=\left[\frac{\ln \alpha \sqrt{\frac{E_{g}}{2 k T}}-\frac{\Phi_{B . i}}{k T}}{\ln \alpha \sqrt{\frac{E g}{2 k T}}-\frac{\Phi_{B . i}}{k T}-\frac{\Phi_{B . f}-\Phi_{B . i}}{k T}}\right]^{\frac{1}{\beta}}=\left[\frac{1}{1-\frac{\Delta \Phi_{B}}{k T\left(\ln \alpha \sqrt{\left.\frac{E g}{2 k T}-\frac{\Phi_{B . i}}{k T}\right)}\right.}}\right]^{\frac{1}{\beta}}
\end{aligned}
$$

It is clear from Equation 7, that the SB height change is related to the change in $\Phi_{B}$. With a reasonable approximation, Equation 8 can be expressed as: $\exp \left[\frac{\Delta \Phi_{S B}}{k T}\right]=\left(1-b \theta_{M}\right)^{-\frac{1}{\beta}}$, Where b is a constant dependent $\propto \frac{\Delta \Phi_{B}}{k T\left(\ln \alpha \sqrt{\frac{E g}{2 k T}}-\frac{\Phi_{B . i}}{k T}\right)}$, and $\theta_{M}$ is the metal surface coverage given below. $\theta_{M}=\left(1-e^{-k_{M} P t}\right), \quad \theta_{N T}=\left(1-e^{-k_{N T} P t}\right), \quad \theta_{M}=\frac{K_{M} P}{K_{M} P+1}, \quad \theta_{N T}=\frac{K_{N T} P}{K_{N T} P+1} . \quad$ The surface coverage, $\theta_{\mathrm{M}}$ and $\theta_{\mathrm{NT}}$ are obtained from the Langmuir model [6][7]. The polarizability and dipole moment of the gas molecule are determined whether $b$ is positive or negative, which corresponds to either a decrease or increase in conductance. The resistance change includes two components: the contact resistance $\left(\mathrm{R}_{\mathrm{M}}\right)$ and the CNT channel resistance $\left(\mathrm{R}_{\mathrm{CNT}}\right)$. Assuming that the initial and final resistances are $R_{0}=\left(R_{M}+R_{V N T}\right)$ and $R_{f}=R_{0=M . f}+R_{C N T . f}$. Then the CNT resistance is inversely proportional with the carrier density $\left(R_{C N T} \propto \frac{1}{n 0}\right)[5,9]$.

$$
\begin{gathered}
\Delta R=R_{f}-R_{0}=R_{M}\left(\frac{R_{M . f}}{R_{M}}-1\right)+R_{C N T}\left(\frac{R_{C N T . f}}{R_{C N T}}-1\right)=R_{M}\left[\frac{n_{0}}{n_{f}} e^{\frac{\Delta \Phi_{S B}}{k_{B} T}}-1\right]+R_{C N T}\left[\frac{n_{0}}{n_{f}}-\right. \\
1]=R_{M}\left[\left(\delta \theta_{N T}+1\right)^{-1}\left(1-b \theta_{M}\right)^{-\frac{1}{\beta}}-1\right]+R_{C N T} \frac{-\delta \theta_{N T}}{\delta \theta_{N T}+1} \\
\frac{\Delta G}{G_{0}}=\frac{G_{f}-G_{0}}{G_{0}}=\frac{G_{f}}{G_{0}}-1=\frac{R_{0}}{R_{f}}-1=\frac{R_{0}-R_{f}}{R_{f}}=\frac{-\Delta R}{R_{0}+\Delta R}=\frac{\left[1-\left(\delta \theta_{N T}+1\right)^{-1}\left(1-b \theta_{M}\right)^{-\frac{1}{\beta}}\right] R_{M}+R_{C N T} \frac{\delta \theta_{N T}}{\delta \theta_{N T}+1}}{\left(\delta \theta_{N T}+1\right)^{-1}\left(1-b \theta_{M}\right)^{-\frac{1}{\beta}} R_{M}+R_{C N T} \frac{1}{\delta \theta_{N T}+1}}
\end{gathered}
$$

$\mathrm{G}$ is the conductance.

\section{WIRELESS TRANSMISSION}

In this section, the complete wireless transmission based on BBB (developed by N.I.) and the Bluetooth techniques were introduced.

\subsection{Hardware Board System}

The assembly of the hardware system consists of three main components: BBB board, Bluetooth chip, and the control unit. The micro USB port of BBB simply works as a power source port; the micro HDMI port was linked to a monitor; the micro SD card was attached to the reader, which worked as a hard disk to the system. The standard USB port was interfaced to the USB hub and then attached to the USB based Bluetooth chip, and the mouse and keyboard adapter. Figure 6 
shows the BBB board used in this work. In this application, the BBB board functions as a mini $\mathrm{PC}$ that communicates with the Bluetooth system. It receives the data from the Labview software and transmits it to another Bluetooth device.

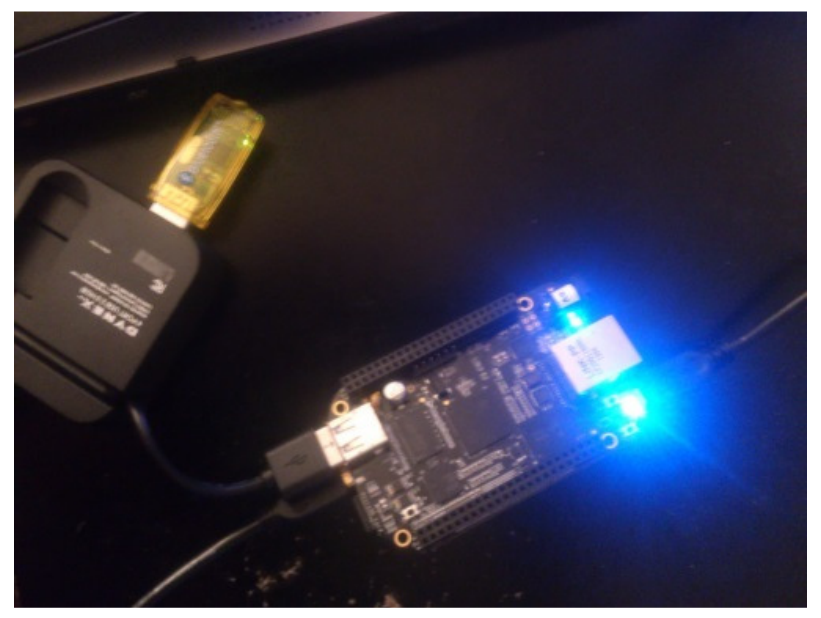

Figure 6: Connection Diagram of BBB Board.

\section{RESULTS \& DISCUSSIONS}

In this section, we report simulation results from Labview for the integrated sensor system, including the temperature, gas, and pressure sensors. The wireless transmission for the dynamic responses of the sensors was verified. The simulation here presents data for one sensor as sample data for the multisensory system.

\subsection{The Temperature Sensor}

The range of the temperature used for testing the sensor was from 283.15 to $353.15 \mathrm{~K}$, which corresponds to 10 and $80{ }^{\circ} \mathrm{C}$, respectively. This range was chosen in order to perform linear response with the CNT resistance. Figure 7 shows the details of the response following the mathematical model. As it can be seen, the curve fits linearly well within the temperature range of 283 to $353 \mathrm{~K}$, and beyond this range, the response is not linear. This is the useful range of Bio and HVAC applications. The $\mathrm{x}$ axles in Figure 7 are time (s). The resistance is changing from 75 to $700 \mathrm{k} \Omega$ and the sensitivity range of the CNT pressure sensing part is $0 \sim 8400 \mathrm{kPa}$. Figure 7 describes the resistance-temperature direct relationship. The slope of the curve (within the desired range of temperature) gives the sensitivity determined by $\Delta \mathrm{R} / \Delta \mathrm{T}$. In this case, the sensitivity is $0.7 \mathrm{k} \Omega / \mathrm{K}$. This indicates that the sensor can detect as small as milli $\mathrm{K}$ change, since the corresponding change in resistance is still in the $10 \mathrm{~s} \mathrm{k} \Omega$ range, and this can be easily detected by the processing unit of the system. 
Temperature

resistance
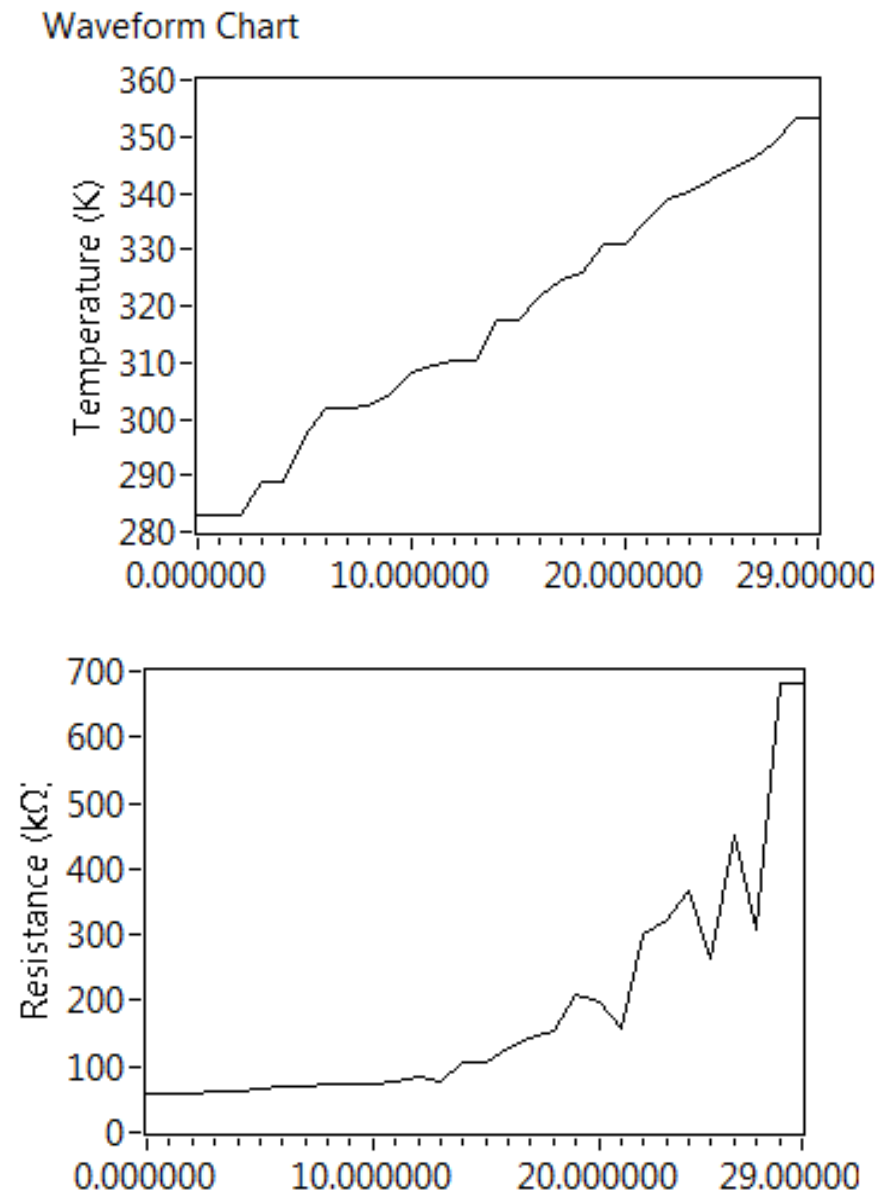

Figure 7: The Simulation Result of the Temperature Sensor.

\subsection{Discussions}

The design of the integrated sensor system built on the same chip, detecting temperature, gases, and pressure that interfere with each other is demonstrated. The nanodevices utilized CNT smart nanomaterials that are appropriate for bio-engineering applications as evidenced by the power level have been determined. The range of detection is determined from the linear part of the sensor characteristics. The error factor from interference was compensated within the DSP unit. The proposed system can be expanded to include more sensors such as liquid and gas flow or blood sugar detectors. For the temperature sensor, an indirect detection method was introduced. The CNTs worked as the pressure sensor to detect the volume change of the thermal expansion elements to avoid the gas influence. In this case, a read-out circuit was to be interfaced to the sensor in order to process the data.

For the gas sensor, the CNTs coating with SS-DNA device was given. The two sequences of SSDNA made the CNTs significantly more sensitive to the gas. To raise the accuracy of the sensor, the twin-sensor with both sequence 1 and sequence 2 SS-DNA coated CNTs was used. The DSP chip could be modified to incorporate both sequence 1 and sequence $2 \% \Delta \mathrm{I} / \mathrm{I}$ data, into the table look up. For the pressure sensor, the CNTs placed between source and drain of the CMOS device 
was introduced. To reducing the effect of the temperature and gas, the CNTs were coated with Parylene C material.

\section{REFERENCES}

[1] Christofer Hierold, CONCEPTS FOR CARBON NANOTUBE SENSORS, Micro and Nanosystems, ETH Zurich.

[2] Verma, R. ; Dept. of Electr. \& Comput. Eng., Indiana Univ.-Purdue Univ. Indianapolis (IUPUI), Indianapolis, IN, USA ; Said, K. ; Salim, J. ; Kimathi, E. Carbon nanotube-based microstrip antenna gas sensor, Circuits and Systems (MWSCAS), 2013 IEEE 56th International Midwest Symposium on, 4-7 Aug. 2013.

[3] M. Fujioka, H. Watanabe, Y. Martin, M. Nakano, Separation and enrichment of semiconducting carbon nanotubes and its application to highly sensitive carbon nanotube gas sensor, 2011 IEEE Nanotechnology Materials and Devices Conference.

[4] Cheng Yung Kuo, Chia Lang Chan, Chie Gau, Chien-Wei Liu, Shiuan Hua Shiau, and Jyh-Hua Ting, Nano Temperature Sensor Using Selective Lateral Growth of Carbon Nanotube Between Electrodes, IEEE TRANSACTIONS ON NANOTECHNOLOGY, VOL. 6, NO. 1, JANUARY 2007.

[5] Cosmin Roman, Florin Ciontu, Bemard Courtois, Single molecule detection and macromolecular weighting using an all-carbon-nanotube nanoelectromechanical sensor, 2004 4th IEEE Conference on Nanotechnology.

[6] Carmen K. M. Fung, Maggie Q. H. Zhang, Rosa H. M Chan and Wen J. Li, A PMMA-BASEDM ICROP RESSURE SENSOR CHIP USING CARBON NANOTUBEASSSENSINGEL EMENTS, Centre for Micro and Nano Systems, The Chinese University of Hong Kong, Hong Kong SAR.

[7] Brendan Crawford, Dan Esposito, David Pelletier, Vishal Jain, Flexible Carbon Nanotube Based Temperature Sensor for Ultra-Small-Site Applications.

[8] Suehiro, G. Zhou and M. Hara, Fabrication of a carbon nanotube-based gas sensor using dieiectrophoresis and its application for ammonia gas sensor using dieiectrophoresis and its application for ammonia detection by impedance spectroscopy, Journal of Physics D, Vol. 36, pp. 109-114 (2003).

[9] Sun Penghua, "MS Thesis" Department of ECE, Purdue School of Engineering and Technology, May 2014. 